\title{
Application of Agglomerative Hierarchical Clustering for Clustering of Time Series Data
}

DOI:

10.1109/ISGT-Europe47291.2020.9248759

10.1109/isgt-europe47291.2020.9248759

Link to publication record in Manchester Research Explorer

\section{Citation for published version (APA):}

Radovanovic, A., Li, J., Milanovic, J. V., Milosavljevic, N., \& Storchi, R. (2020). Application of Agglomerative Hierarchical Clustering for Clustering of Time Series Data. In Proceedings of 2020 IEEE PES Innovative Smart Grid Technologies Europe, ISGT-Europe 2020 (pp. 640-644). (IEEE PES Innovative Smart Grid Technologies Conference Europe; Vol. 2020-October). IEEE. https://doi.org/10.1109/ISGT-Europe47291.2020.9248759, https://doi.org/10.1109/isgt-europe47291.2020.9248759

Published in:

Proceedings of 2020 IEEE PES Innovative Smart Grid Technologies Europe, ISGT-Europe 2020

\section{Citing this paper}

Please note that where the full-text provided on Manchester Research Explorer is the Author Accepted Manuscript or Proof version this may differ from the final Published version. If citing, it is advised that you check and use the publisher's definitive version.

\section{General rights}

Copyright and moral rights for the publications made accessible in the Research Explorer are retained by the authors and/or other copyright owners and it is a condition of accessing publications that users recognise and abide by the legal requirements associated with these rights.

\section{Takedown policy}

If you believe that this document breaches copyright please refer to the University of Manchester's Takedown Procedures [http://man.ac.uk/04Y6Bo] or contact uml.scholarlycommunications@manchester.ac.uk providing relevant details, so we can investigate your claim.

\section{OPEN ACCESS}




\section{Application of Agglomerative Hierarchical Clustering for Clustering of Time Series Data}

\author{
Ana Radovanović, Student Member \\ IEEE \\ Department of Electrical and Electronic \\ Engineering \\ The University of Manchester \\ Manchester, UK
ana.radovanovic@manchester.ac.uk \\ Manchester, UK
ana.radovanovic@manchester.ac.uk
}

\author{
Nina Milosavljević \\ Division of Neuroscience and Experimental Psychology \\ The University of Manchester \\ Manchester, UK \\ nina.milosavljevic@manchester.ac.uk
}

\author{
Jovica V. Milanović, Fellow IEEE \\ Department of Electrical and Electronic \\ Engineering \\ The University of Manchester \\ Manchester, UK \\ milanovic@manchester.ac.uk
}

\begin{abstract}
The advancements in technology have made it possible to automatically record and store large amount of data, which has resulted in a need for development and application of efficient data analysis techniques. Unsupervised data clustering methods have proven to be capable of extracting useful information from various types and sizes of datasets. This paper investigates the performance of the standard agglomerative hierarchical clustering algorithm using two time series datasets from electric power system and neuroscience area. The main steps in clustering procedure are presented in detail. Results show that the effectiveness of the clustering algorithm is affected to a large extent by the main characteristics of the clustering data and algorithm's parameters.
\end{abstract}

Keywords-clustering, electric power system, hierarchical clustering algorithm, neuroscience

\section{INTRODUCTION}

With ever growing capabilities for data collection and storage, data mining techniques are becoming extremely useful for efficient modelling and analysis of various systems. One of the widely used data mining methods is data clustering, which represents unsupervised grouping of different objects according to their similarity. The objective is to achieve high similarity between objects belonging to the same group, i.e., cluster, and high dissimilarity between objects from different clusters [1].

Due to increased interest in temporal data analysis in various areas, time series data clustering is gaining popularity [2], [3]. Even though the characteristics of time series data are considerably different from the ones of static data (data whose characteristics do not change with time), clustering of both data types is usually carried out using the same clustering techniques. This is mainly because most of the existing clustering algorithms were originally developed for static data, as this data type was in the focus of data clustering in the past [2]. As temporal sequences are characterized by high dimensionality in space, clustering algorithms designed for static data are usually slightly modified in order to be used for time series clustering. So far, two methods have been used for addressing the issue. One approach, so-called raw data-based approach, is based on combining traditional clustering algorithms with distance measures that are more suitable for finding similarity in time. The other method attempts to

\author{
Riccardo Storchi \\ Division of Neuroscience and Experimental Psychology \\ The University of Manchester \\ Manchester, UK \\ riccardo.storchi@manchester.ac.uk
}

reduce the dimensionality of a clustering task by representing temporal sequences by a set of their most important features or a certain mathematical model. However, this approach is highly application dependent [2], [3].

Over the years a large number of clustering algorithms suitable for various clustering problems have been developed. However, the basic clustering algorithms, partitioning (among them the most popular are the k-means and the k-medoids) and hierarchical algorithms, have been most commonly utilized [2]. The simplicity and availability in software packages are the major reasons for their widespread application [1], [4].

In this research both of these methods were tested namely, the k-medoids, as one of the most widely used partitioning algorithms, and the agglomerative hierarchical algorithm to assess their suitability for clustering time series data from different fields of study. This paper presents the results of the assessment of the adequacy of using standard agglomerative hierarchical algorithm for time series data clustering. The focus is on raw data-based approach. All the main stages of clustering process are investigated in detail. Two distinct time series datasets from electrical power system and neuroscience area are used for testing the effectiveness of the selected clustering algorithm.

\section{Clustering Procedure}

\section{A. Hierarchical Clustering Algorithm}

Hierarchical clustering (HC) algorithms perform clustering by organizing objects into a "hierarchical" structure [2], [5]. Depending on the way the structure is obtained, they can be divided into the agglomerative (bottom-up) and divisive (top-down) hierarchical algorithms. The first approach considers each clustering object as an individual cluster at the beginning of clustering process, and then iteratively merges the objects into one cluster. On the other hand, divisive algorithms treat all the objects as one cluster in the beginning and divide it into multiple clusters iteratively afterwards. Once merging/splitting of clusters has been carried out, it is not possible to make corrections at later stages of the clustering procedure [2], [5]. The computational complexity for bottom-up approach is $O\left(N^{2}\right)$, where $N$ is the number of clustering objects, while top-down algorithms have 
much higher computational complexity, $O\left(2^{(N-1)}\right)$. In this paper the focus is on the agglomerative $\mathrm{HC}$ algorithm.

The process of merging/splitting of clusters is usually presented in the form of a clustering tree (so-called dendrogram). An example of a dendrogram for bottom-up HC algorithm is shown in Fig. 1. Clusters are represented by nodes, whereas the vertical axis of the dendrogram represents the similarity measure between clusters calculated based on the chosen linkage and distance measure. Linkage measure defines how the similarity between clusters is assessed and the most commonly used ones are: single, complete and average (weighted and unweighted) [4]. Single and complete linkage measures take into account only the distance between the closest and farthest elements in clusters, respectively, whereas average linkage measures are based on the average distance between elements in two clusters. The weighted average linkage takes into consideration the number of elements in clusters as well.

When it comes to distance measures, the most widely used distance measures in time series clustering are the Euclidean, Pearson's correlation, Spearman and Dynamic Time Warping (DTW) distance [2], [7]. The expressions for calculating the Euclidean, Pearson's correlation and Spearman distances are as follows:

$$
\begin{gathered}
d_{\text {Euclidean }}=\sqrt{\sum_{i=1}^{n}\left(x_{i}-y_{i}\right)^{2},} \\
d_{\text {Pearson }}=1-\frac{\sum_{i=1}^{n}\left(x_{i}-\bar{x}\right)\left(y_{i}-\bar{y}\right)}{\sqrt{\sum_{i=1}^{n}\left(x_{i}-\bar{x}\right)^{2}} \sqrt{\sum_{i=1}^{n}\left(y_{i}-\bar{y}\right)^{2}}}, \\
d_{\text {Spearman }}=1-\frac{\sum_{i=1}^{n}\left(r_{x, i}-\overline{r_{x}}\right)\left(r_{y, i}-\overline{r_{y}}\right)}{\sqrt{\sum_{i=1}^{n}\left(r_{x, i}-\overline{r_{x}}\right)^{2}} \sqrt{\sum_{i=1}^{n}\left(r_{y, i}-\overline{r_{y}}\right)^{2}}},
\end{gathered}
$$

where $x$ and $y$ are time series, $n$ is the number of data samples, $\bar{x}$ and $\bar{y}$ are the mean values of $x$ and $y, r_{x}$ and $r_{y}$ are rank vectors of $x$ and $y$, and $\overline{r_{x}}$ and $\overline{r_{y}}$ are the mean values of $r_{x}$ and $r_{y}$. These three distances are so-called the lock-step distance measures as they consider distances between the samples occurring at the same time steps.

The DTW distance is based on finding the minimum warping path between the analyzed temporal sequences. The warping path is equal to the sum of Euclidean distances between samples in time series, whereby these samples do not necessarily have to occur at the same time steps. Unlike the lock-step distances, the DTW distance is capable of dealing with time series of different length. However, the computational complexity of the DTW distance is high $(O(m n)$, where $m$ and $n$ are the lengths of time sequences) [8].

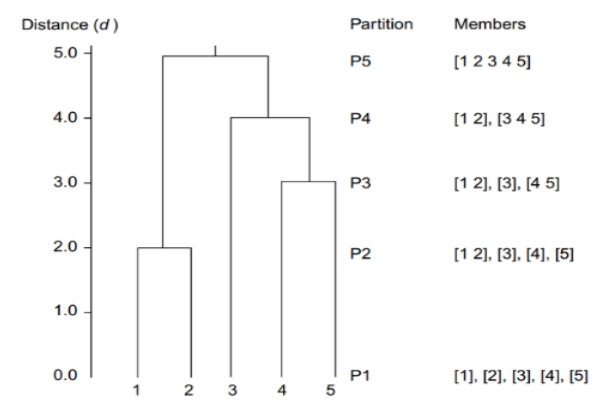

Fig. 1. Graphical representation of a dendrogram for bottom-up HC algorithm (adopted from [6]).

\section{B. Selection of the Optimal Number of Clusters}

Even though the number of clusters does not have to be defined prior to carrying out data clustering, the problem of determining the optimal number of clusters, that is, a height level at which the dendrogram should be cut, still remains. The cut-level defines the minimum distance between obtained clusters, and objects that are grouped together below the cut-level are considered to be similar to each other [6]. Clustering evaluation indices are commonly used to address this problem. Namely, the algorithm is repeated for a range of the number of clusters and the selected clustering index is calculated for each clustering result. The optimal number of clusters corresponds to the value at which the clustering evaluation index has the optimal value. Some of the clustering indices commonly used for the estimation of the optimal number of clusters are as follows:

- Mean square error (MSE): The MSE index calculates the average distance between a clustering object and its corresponding cluster representative [5], [9]:

$$
M S E=\frac{1}{N} \sum_{i=1}^{N} d^{2}\left(x_{i}, w_{j: x_{i} \in \Omega_{j}}\right),
$$

where $x_{i}$ is the $i$-th clustering object, $w_{j}$ is a representative of the $j$-th cluster, $\Omega_{j}$ is a set of clustering objects belonging to the $j$-th cluster, $d()$ is a distance measure. A cluster representative is defined as an average of cluster elements.

- Clustering dispersion index (CDI): The CDI is defined as a ratio of the average distance between the objects within the same cluster and the average distance between the representatives of clusters [5], [6], [9]:

$$
\begin{gathered}
C D I=\frac{\left(\frac{1}{k} \sum_{j=1}^{k} \bar{d}^{2}\left(\Omega_{j}\right)\right)}{\bar{d}(W)}, \\
\bar{d}^{2}\left(\Omega_{j}\right)=\frac{1}{2 N_{j}} \sum_{x_{i} \in \Omega_{j}} d^{2}\left(x_{i}, \Omega_{j}\right), \\
d^{2}\left(x_{i}, \Omega_{j}\right)=\frac{1}{N_{j}} \sum_{y \in \Omega_{j}} d^{2}\left(x_{i}, y\right),
\end{gathered}
$$

where $k$ is the number of clusters, $W$ is a set of cluster representatives, $y$ is an element of the $j$-th cluster, $N_{j}$ is the number of elements in the $j$-th cluster.

- Within cluster sum of squares to between cluster variation ratio (WCBCR): The WCBCR is calculated on the basis of the distances between each object and its cluster representative and the distances between cluster representatives [9]:

$$
W C B C R=\frac{\sum_{j=1}^{k} \sum_{x_{i} \in \Omega_{j}} d^{2}\left(x_{i}, w_{j}\right)}{\sum_{1 \leq q<p}^{k} d^{2}\left(w_{p}, w_{q}\right)} .
$$

Lower values of all afore-mentioned indices imply better clustering results. The optimal number of clusters is determined using the elbow method [5]. The idea of the method is to plot the values of the clustering index against the number of clusters and the optimal number of clusters corresponds to the point where the curve reaches its knee. The 


\section{ACCEPTED VERSION OF THE PAPER}

knee of a curve is commonly estimated using the two-tangent technique presented in [9].

\section{RESULTS AND DISCUSSION}

The agglomerative $\mathrm{HC}$ algorithm with weighted average linkage measure is tested on two types of time series datasets from electric power system and neuroscience area. Weighted average linkage measure is selected for time series clustering as it represents a compromise between two extremes, single and complete linkage. Four distance measures (the Euclidean, Pearson's correlation, Spearman and DTW) and three clustering evaluation indicators (MSE, CDI and WCBCR) are used for assessing the performance of the algorithm. Data processing and clustering procedures were performed in Matlab R2019a software package [10].

\section{A. Case Study I: Power System Dataset}

The test dataset consists of active power transient responses of a hybrid renewable energy source (HRES) plant at the point of common coupling. The HRES plant consists of a wind farm, a photovoltaic, hydro, biomass and biogas plant and battery energy storage system. A three-phase self-clearing fault with a duration of $100 \mathrm{~ms}$ was selected as an external system disturbance. The total number of 1,600 plant operating conditions was analyzed. The responses were recorded for a period of $9 \mathrm{~s}$ after the fault clearing and with a sampling rate of $10 \mathrm{~ms}$. All analyzed responses are stable and in the form of damped electromechanical oscillations. In order to identify similarities in shape, the active power responses had to be normalized prior to carrying out clustering procedure as steady state power output of the hybrid plant was not the same in all case studies. Standard z-normalization was used for that purpose [11]:

$$
z(t)=\frac{P(t)-P_{\text {mean }}}{S D(P(t))},
$$

where $z(t)$ is a z-normalized power response in p.u, $P(t)$ is a power response in $\mathrm{MW}, P_{\text {mean }}$ is the average of the whole response, $S D(P(t))$ is a standard deviation of the response.

The change of clustering indices with the number of clusters for four distance measures is shown in Fig. 2 - Fig. 4. As distance measure determines how the similarity between clustering objects is calculated, different distances produce different curves for the same clustering index. The Spearman distance results in the most regular L-shaped curves, whereas the knee of curves produced by the Euclidean and Pearson's correlation distances is noticeable in the case of the WCBCR index only. As the curves for the WCBCR index are characterized by the most noticeable knee for all considered distances, the index is chosen for estimating the optimal number of clusters in the active power dataset. According to this index, the optimal number of clusters in the case of Euclidean and Pearson's correlation distances is four, while three clusters are required for the other two distance measures.

Distance measures characterized by the same optimal number of clusters result in identical clustering results. All outputs of the HC algorithm for the Euclidean and Pearson's correlation distances are presented in Fig. 5 and Fig. 6. Cluster 1 in Fig. 5 is a common cluster for all considered distances, which means that all distance measures identify a group of highly damped responses successfully. Also, this group represents the best cluster in terms of compactness and separation from other clusters for all four distance measures.
When it comes to the remaining clusters, there are certain differences in characteristics of the responses belonging to the same cluster. As shown in Fig. 6, clusters 2 and 3 in the case of the Euclidean and Pearson's correlation distances consist of responses having different phases, damping and amplitude. As for the remaining clusters produced by the Spearman and DTW distances, they can be obtained on the basis of the clusters shown in Fig. 6. Namely, one of the remaining two clusters corresponds to cluster 3 in Fig. 6, whereas the other one is a combination of clusters 2 and 4 in Fig. 6.

As clustering quality of the Euclidean and Pearson's correlation distances is slightly higher than that of the other two distance measures, and their computational complexity is much lower compared to the DTW distance, the Euclidean and Pearson's correlation distances can be considered as the optimal distance measures for the clustering of active power responses.
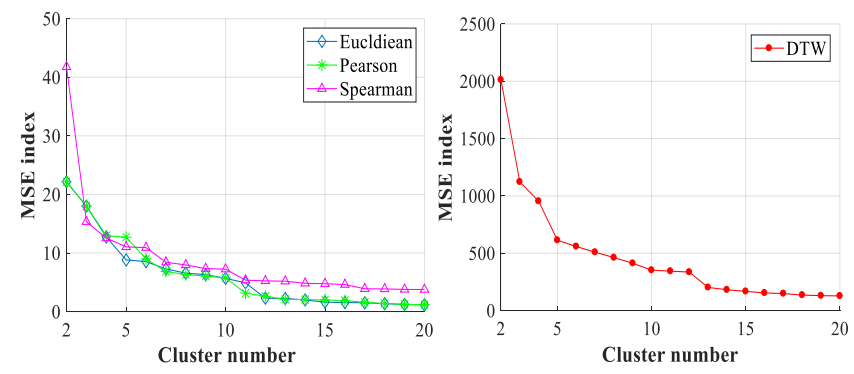

Fig. 2. Power system dataset: MSE index for the analyzed distance measures.
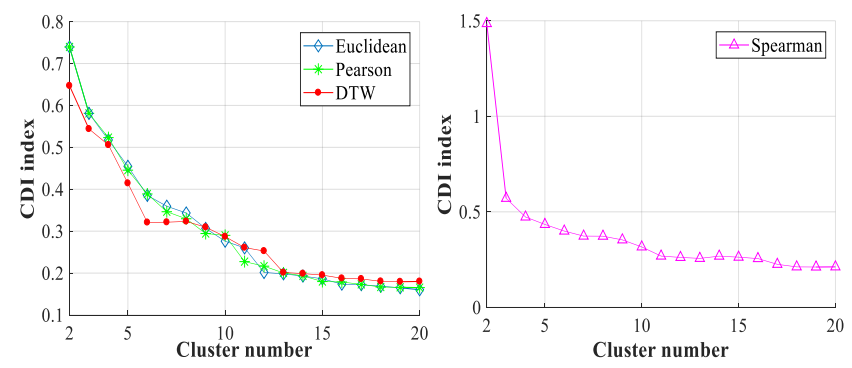

Fig. 3. Power system dataset: CDI index for the analyzed distance measures.
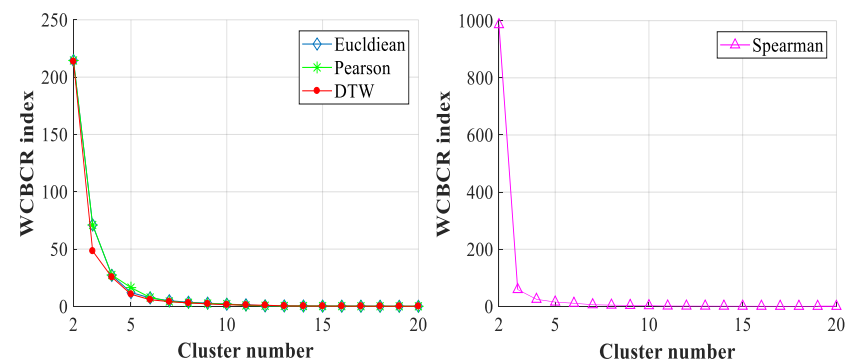

Fig. 4. Power system dataset: WCBCR index for the analyzed distance measures.

\section{B. Case Study II: Neuroscience Dataset}

The second case study concerns the data about the responses of 1,492 neurons to light stimuli, so-called spike-train data as a neuron reacts to a stimulus in the form of a spike (impulse) (data taken from [13]). A clustering object is a sequence of 80 trials, where each trial represents an exposure of the neuron to the stimulus for a period of 10.99 seconds. Neuron reactions to the stimulus were recorded as spike frequencies in 10-milisecond intervals (number of 
spikes in the interval divided by the interval size). In this way, a large dataset of size $1,492 \times(80 \times 1,099)$ was created. Unlike plant power responses analyzed in case study I, spike-train data are in the form of impulse sequences with a large number of zeros corresponding to neuron inactivity to light stimuli.

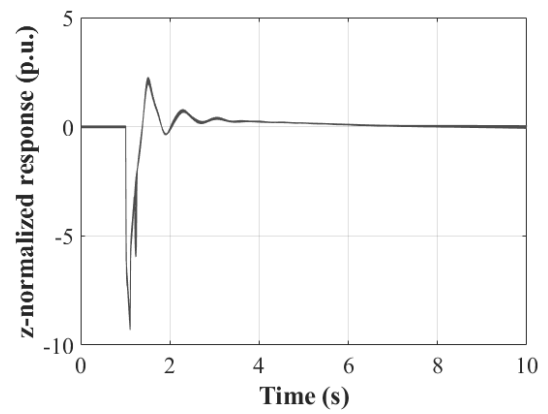

Fig. 5. Cluster 1 for all considered distance measures.
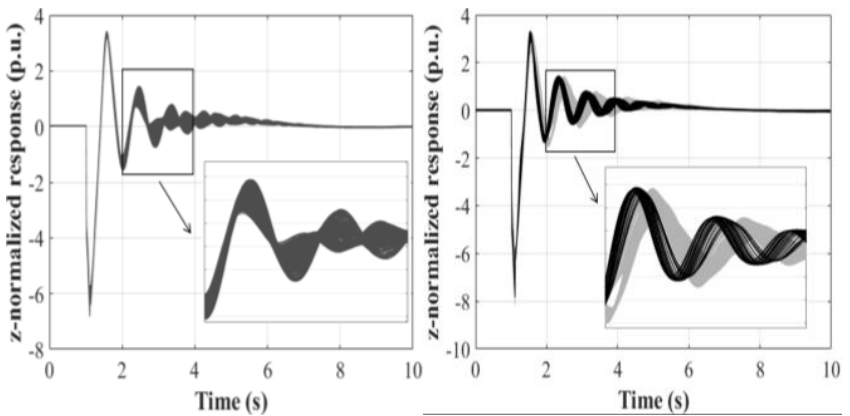

Fig. 6. Cluster 2 (left), Cluster 3 (right - light grey) and Cluster 4 (right black) in the case of the Euclidean and Pearson's correlation distances.

As the computational complexity of the $\mathrm{HC}$ algorithm is not negligible, the dataset size had to be reduced prior to carrying out the clustering process. Thus, for each neuron, a sequence of 80 trials was split into 2 groups corresponding to the first and last 40 trials, as there is a clear distinction between the characteristics of these trials. Then, the trials belonging to the same group were summed. This resulted into a significant dataset size reduction - the new dataset size is $1,492 \times(2 \mathrm{x}$ 1,099). Another issue with the recorded data is that they contain a large number of zeros corresponding to neuron inactivity to light stimuli. As this can cause problems when calculating distances between clustering objects (e.g., the denominator in (2) would be equal to zero in case there is no neuron activity during a trial), the data were Gaussian filtered (the parameters of Gaussian filter are $L=30, \sigma=3$ ). The final step of data pre-processing was z-normalization, as the clustering task is finding similarity in firing patterns, similarly to the first case study.

The curves representing the change of the analyzed clustering evaluation indices with the number of clusters are shown in Fig. 7 and Fig. 8. Unlike in the case of the power transient responses, the MSE index is unsuitable for the estimation of the optimal number of clusters as it is characterized by non-L-shaped curves for all distances. As in the previous case study, the WCBCR index has the most noticeable knee; thus, this indicator is used in data clustering process. The optimal number of clusters for the DTW distance is three, whereas four clusters are optimal for the lock-step distance measures.

Unlike in case study I, different distance measures produce different clustering results. However, clustering quality is not satisfactory for any of the considered distances as almost all obtained clusters are heterogeneous, i.e., they contain neurons with different firing patterns. The lowest level of compactness is obtained in the case of the Spearman distance measure, whereas the clustering quality of the best and worst clusters (in terms of compactness and separation from other clusters) of the remaining distances is similar. As the worst cluster in the case of the DTW distance contains very small number of neurons (around 70), the DTW distance can be regarded as the most suitable for the spike-train data clustering.

Fig. 9 - Fig. 12 illustrate the worst clusters produced by each of the distance measures, while an example of the best clusters is shown in Fig. 13. Non-normalized data are used for presenting clustering results, as z-normalized spike-train data are not suitable for visualization of clustering results.
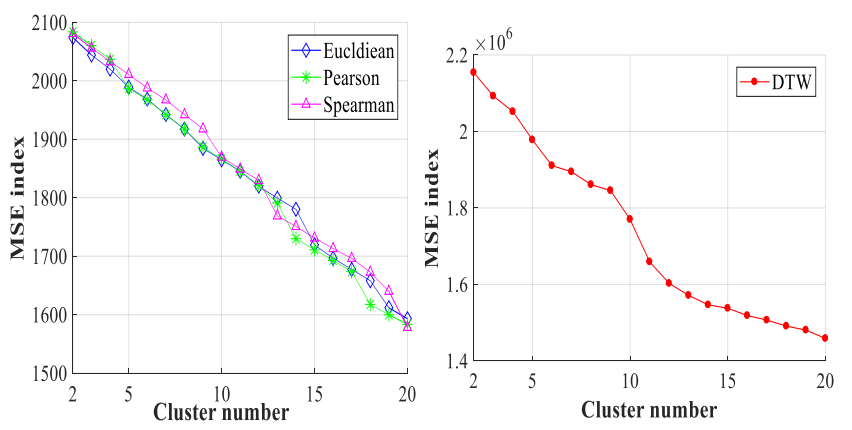

Fig. 7. Neuroscience dataset: MSE index for the analyzed distance measures.
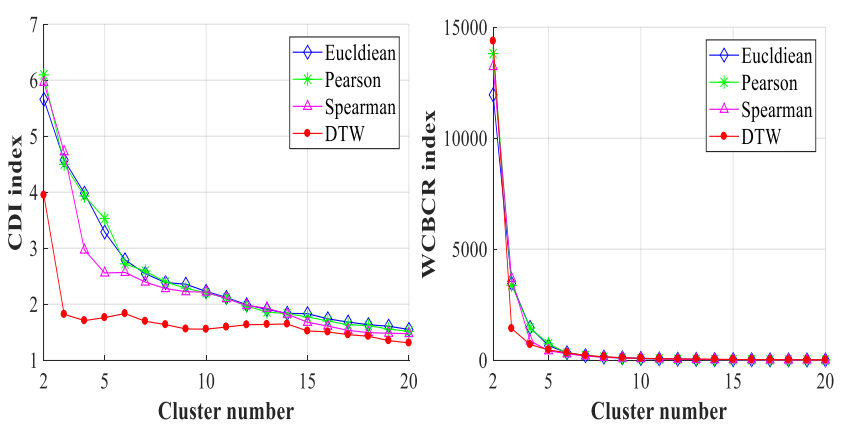

Fig. 8. Neuroscience dataset: CDI and WCBCR indices for the analyzed distance measures.
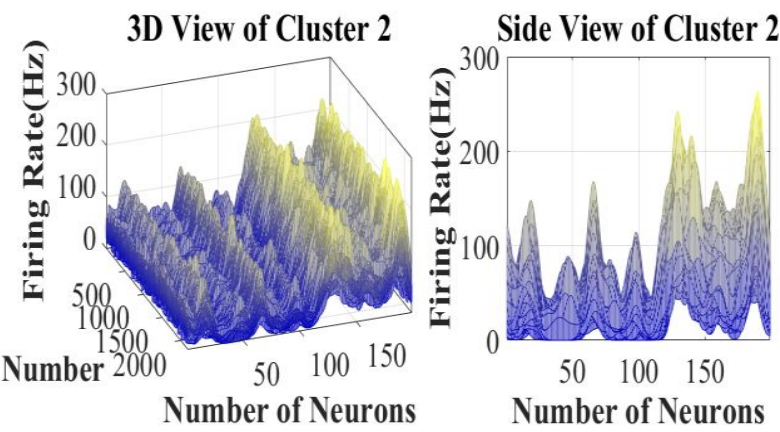

Fig. 9. Neuroscience dataset: 3D view (left) and side view (right) of the worst cluster in the case of the Euclidean distance. 


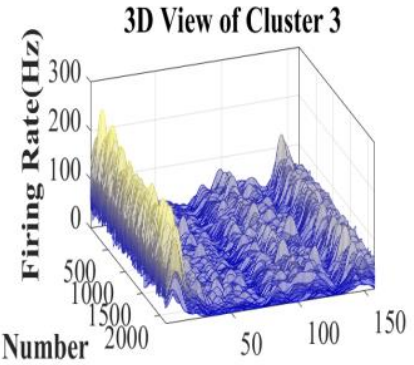

Number of Neurons

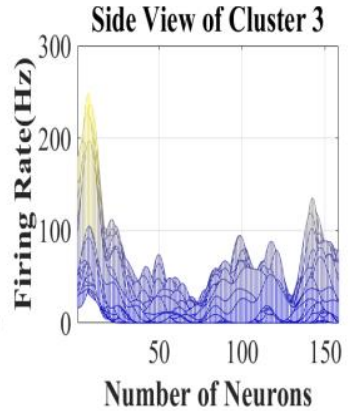

Number of Neurons

Fig. 10. Neuroscience dataset: 3D view (left) and side view (right) of the worst cluster in the case of the Pearson's correlation distance.

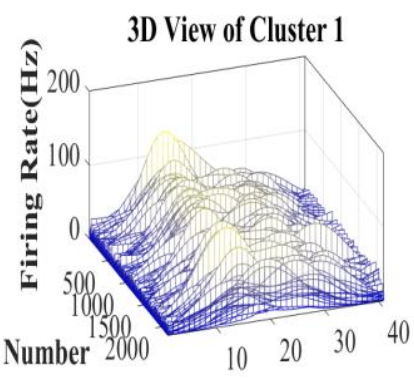

Number of Neurons

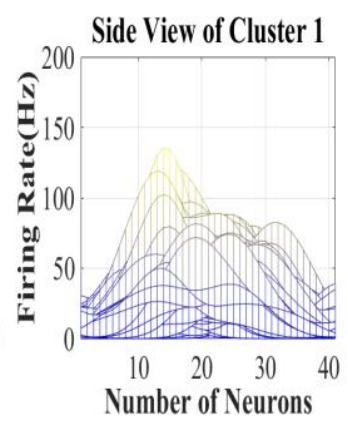

Fig. 11. Neuroscience dataset: 3D view (left) and side view (right) of the worst cluster in the case of the Spearman distance.
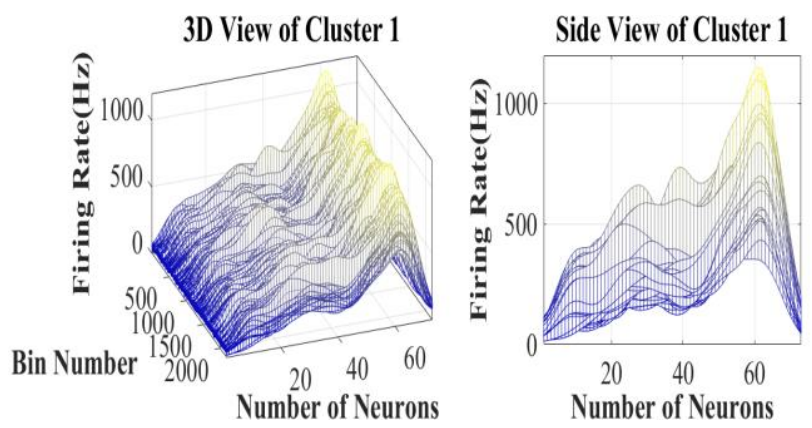

Fig. 12. Neuroscience dataset: 3D view (left) and side view (right) of the worst cluster in the case of the DTW distance.

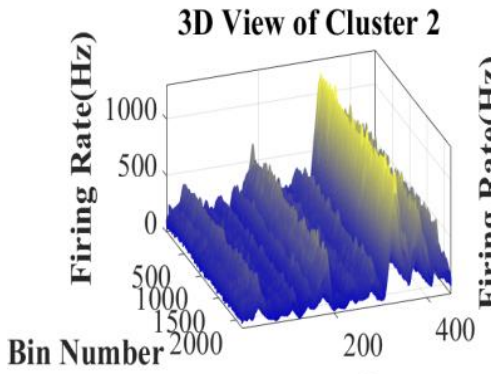

Number of Neurons
Side View of Cluster 2

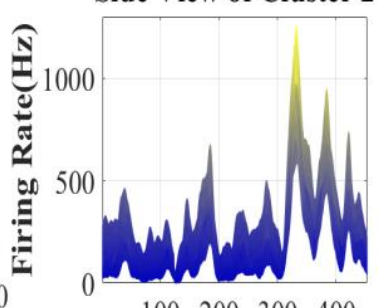

100200300400

Number of Neurons
Fig. 13. Neuroscience dataset: 3D view (left) and side view (right) of the best cluster in the case of the DTW distance.

\section{CONCLUSION}

The paper explores the suitability of the bottom-up HC algorithm for time series clustering using power system and neuroscience data. Results have demonstrated that the performance of the $\mathrm{HC}$ algorithm is affected by dataset characteristics to a large extent. Different distances have shown to be more suitable for different datasets. The Euclidean and Pearson's correlation distances are the most adequate for active power responses of the hybrid power plant, whereas the DTW distance is the most appropriate for the spike-train data.

When it comes to the selection of the optimal number of clusters, the three considered clustering evaluation indices suggest different numbers of clusters for the same distance measure. The WCBCR index has shown to be the most robust to the choice of distance measure and clustering dataset, while the CDI and MSE indices are characterized by irregular-shaped curves.

Even though the agglomerative $\mathrm{HC}$ algorithm (as an example of hierarchical algorithms) and the four distance measures were selected based on comprehensive literature review as the best candidates for the task in hand, the perfectly compact and separated clusters have not been obtained. Therefore, future research will focus on analyzing the suitability of other clustering algorithms and evaluation indices for clustering the time series power system and neuroscience data.

\section{ACKNOWLEDGMENT AND DISCLAIMER}

The research is supported by the EU H2020 project CROSSBOW (grant agreement 773430), Fight for Sight Fellowship 5047/5048 and National Centre for Replacement Refinement and Reduction of Animals in Research (NC3Rs) via a David Sainsbury Fellowship (NC/P001505/1). The paper reflects only the authors' views and neither the Agency nor the Commission are responsible for any use that may be made of the information contained therein.

\section{REFERENCES}

[1] A. K. Jain, M. N. Murty, P. J. Flynn, "Data clustering: a review," ACM Comput. Surv., vol. 31, no. 3, pp. 264-323, September 1999.

[2] T. Warren Liao, "Clustering of time series data - a survey," Pattern Recognit., vol. 38, no. 11, pp. 1857-1874, November 2005.

[3] S. Aghabozorgi, A. Seyed Shirkhorshidi, and T. Ying Wah, "Time series clustering - A decade review," Inform. Syst., vol. 53, pp. 16-38, October - November 2015.

[4] C. C. Aggarwal, C. K. Reddy, Data Clustering: Algorithms and Applications. Florida, USA: CRC Press, 2013.

[5] J. Han, M. Kamber, J. Pei, Data Mining Concepts and Techniques, 3rd ed. Waltam, USA: Elsevier, 2012.

[6] S. L. Everitt, M. Leese, D. Stahl, Cluster analysis, 5ht ed. London, UK: John Wiley \& Sons, Ltd., 2011.

[7] A. Fujita, J. R. Sato, M. A. A. Demasi, M. C. Sgayar, C. E. Ferreira, and S. Miyano, "Comparing Pearson, Spearman and Hoeffding's measure for gene expression association analysis," J. Bioinform. Comput. Biol., vol. 7, no. 4, pp. 663-684, August 2009.

[8] Y. S. Jeong, M. K. Jeong, O. A. Omitaomu, "Weighted dynamic time warping for time series classification," Pattern Recognit., vol. 44, no. 9, pp. 2231-2240, September 2011.

[9] G. J. Tsekouras, P. B. Kotoulas, C. D. Tsirekis, E. N. Dialynas, and N. D. Hatziargyriou, "A pattern recognition methodology for evaluation of load profiles and typical days of large electricity customers," Electr. Pow. Syst. Res., vol. 78, no. 9, pp. 1494-1510, September 2008.

[10] "MATLAB and Statistics Toolbox Release 2019," The Mathworks, Inc., Natick, Massachusetts, United States.

[11] I. B. Mohamad, D. Usman, "Standardization and its effects on k-means clustering algorithm,” Res. J. Appl. Sci. Eng. Tech., vol. 6, no. 17, pp. 3299-3303, September 2013.

[12] G. Chicco, R. Napoli, P. Postolache, M. Scutariu and C. Toader, "Customer characterization for improving the tariff offer," IEEE Trans. on Power Systems, vol. 18, no. 1, pp. 381-387, February 2003.

[13] N. Milosavljević, R. Storchi, C. G. Eleftheriou, A. Colins and R. S. Peterson, "Photoreceptive retinal ganglion cells control the information rate of the optic nerve," Proc. Natl. Acad. Sci. USA, vol. 115, no. 50, pp. E11817-E11826, December 2018. 